\title{
RENDIMENTO DA CANA-DE-AÇÚCAR É AFETADO POR FLÚOR ( ${ }^{\mathbf{1}}$ )
}

\author{
RAFAEL OTTO $\left({ }^{2}\right)$; MARIA REGINA MEIRELES DE FARIA $\left({ }^{3}\right)$; FÁBIO EDUARDO DE CAMPOS QUEIROZ $\left({ }^{3}\right)$; \\ THIAGO AUGUSTO DE MOURA $\left({ }^{3}\right)$; GODOFREDO CESAR VITTI $\left({ }^{* *}\right)$; HEITOR CANTARELLA $\left({ }^{4}\right)$
}

\begin{abstract}
RESUMO
O aquecimento de rochas e solos em indústrias de fundição, siderúrgicas, fertilizantes, vidro e cerâmica liberam compostos contendo flúor (F) na atmosfera. Em área cultivada com duas variedades de cana-de-açúcar no município de Cordeirópolis (SP), adjacente à indústria produtora de cerâmica, foram diagnosticadas queda na produtividade e sintomas de toxidez comparados ao canavial distante da fonte emissora de F. Para a variedade SP80 1816, os teores de F variaram de 56 a $3 \mathrm{mg} \mathrm{kg}^{-1}$ em amostras de folhas coletadas pela técnica da diagnose foliar, ao lado e a $13.000 \mathrm{~m}$ da cerâmica, respectivamente. Para a variedade RB83 5089 o teor de F nas folhas foi de $26 \mathrm{mg} \mathrm{kg}^{-1}$ a $80 \mathrm{~m}$ da cerâmica. Esses teores estão acima dos considerados normais, de $10 \mathrm{mg} \mathrm{kg}^{-1}$ (ou menor) em áreas sem influência de emissão de F. A produtividade média foi de $54 \mathrm{Mg} \mathrm{ha}^{-1}$ na cana planta $\left(1 .^{\circ}\right.$ corte) nas duas variedades e na soqueira $\left(2 .^{\circ}\right.$ corte) de $50 \mathrm{Mg} \mathrm{ha}^{-1}$ para SP80 1816 (mais sensível ao F) e $60 \mathrm{Mg} \mathrm{ha}^{-1}$ para a RB83 5089 (menos sensível ao F). Na área distante $13.000 \mathrm{~m}$ da fonte emissora de F não se observou sintomas de toxidez e a produtividade atingiu $82 \mathrm{Mg} \mathrm{ha}^{-1}$ na cana-planta e $100 \mathrm{Mg} \mathrm{ha}^{-1}$ na soqueira para a variedade SP80 1816. O F emitido por indústria de cerâmica sem uso adequado de filtro parece limitar a produtividade da cana-de-açúcar, fato revelado pela diagnose visual, foliar e queda na produção de colmos. Constatou-se, também, na canade-açúcar sensibilidade aos efeitos causados pelo excesso de F.
\end{abstract}

Palavras-chave: indústria de cerâmica; toxicidade; produtividade.

\section{ABSTRACT \\ YIELD OF SUGAR CANE AS AFFECTED BY FLUORINE}

Heating minerals, roctks, clays and soils in aluminum and iron smelters, and in ceramic, glass, and fertilizer industries release gaseous forms of fluorine into the atmosphere. In an area grown with two varieties of sugar cane (Saccharum officinarum L.) in Cordeirópolis, SP (Brazil) toxicity symptoms and abnormally low yields were observed in areas nearby as compared with those far from a ceramic industry. For the SP80 1816 sugarcane variety the leaf contents of F varied from $56 \mathrm{mg} \mathrm{kg}^{-1}$ of F in the dry matter of leaves collected according to recommended diagnostic techniques near the ceramic industry to $3 \mathrm{mg}$ $\mathrm{kg}^{-1} \mathrm{~F}$ in samples obtained $13.000 \mathrm{~m}$ away. Fluorine content in the RB83 5089 variety was $26 \mathrm{mg} \mathrm{kg}^{-1}$ in samples collected about $80 \mathrm{~m}$ from the ceramic industry. These values are higher than the $10 \mathrm{mg} \mathrm{kg}^{-1} \mathrm{~F}$ (or less) that is considered normal for plants grown in areas not affected by atmospheric $\mathrm{F}$ emissions. Average sugar cane yields of the first cut of the plant cycle were $54 \mathrm{Mg} \mathrm{ha}^{-1}$ for both varieties in the area

( $\left.{ }^{1}\right)$ Recebido para publicação em 10 de agosto e aceito em 12 de abril de 2007.

$\left({ }^{2}\right)$ Mestrando do Programa de Solos e Nutrição de Plantas da ESALQ/USP Bolsista FAPESP. E-mail: rotto@esalq.usp.br.

$\left({ }^{3}\right)$ Departamento de Ciência do Solo, ESALQ/USP, Caixa Postal 9, 13418-900 Piracicaba (SP). Email: gcvitti@esalq.usp.br. (*) Autor correspondente.

$\left({ }^{4}\right)$ Centro de Pesquisa e Desenvolvimento de Solos e Recursos Ambientais, Caixa Postal 28, 13001-970 Campinas (SP). E-mail: cantarella@iac.sp.gov.br. 
close to the ceramic industry. Average yields of the second cut (first ratoon) were $50 \mathrm{Mg} \mathrm{ha}^{-1}$ for the SP 801816 variety (more sensitive to $F$ ) and $60 \mathrm{Mg} \mathrm{ha}^{-1}$ for the RB 835089 variety (less sensitive to F). In the area about $13.000 \mathrm{~m}$ from the industry, where no foliar symptoms of toxicity were observed, yields reached $82 \mathrm{Mg} \mathrm{ha}^{-1}$ in the first cut and $100 \mathrm{Mg} \mathrm{ha}^{-1}$ in the ratoon. Fluorine released by the ceramic industry without adequate gas emission filters seems to limit sugar cane yields as shown by visual symptoms, F concentration in leaves, and yield reduction in the vicinity of the source of $F$. The sugar cane varieties evaluated showed to be sensitive to the effects of excess $\mathrm{F}$ accumulation.

Key words: ceramic industry; toxicity; productivity

\section{Introdução}

O setor cerâmico da região de Santa Gertrudes, no Estado de São Paulo, responde hoje por cerca de $50 \%$ da produção nacional de pisos e cerâmica esmaltada. Quarenta e uma indústrias estão presentes na região contribuindo para o crescimento econômico local, porém trazendo alguns efeitos deletérios para o ambiente (CETESB, 1998). Além da atividade ceramista, a agricultura se destaca na região com culturas anuais, florestais, perenes e cana-deaçúcar.

O aquecimento de rochas e solos em indústrias de fundição de alumínio e outros metais não ferrosos, fertilizantes fosfatados e outros fertilizantes minerais, vidro, cerâmica e siderúrgica liberam compostos contendo flúor na atmosfera (ARNDT et al., 1995).

O flúor é absorvido da atmosfera pelas plantas principalmente pelas folhas. Em áreas sem emissão de flúor as plantas contêm, normalmente, menos de $10 \mathrm{mg} \mathrm{kg}^{-1}$ de $\mathrm{F}$ no tecido vegetal, segundo GARBER at al. (1967) e outros pesquisadores, citados em ARNDT et al. (1995). BREWER et al. (1965) ressaltaram que sintomas podem aparecer em folhas de gladíolo quando os teores de F estão na faixa de 29 a $48 \mathrm{mg}$ $\mathrm{kg}^{-1}$, comprometendo a produção. ForTes et al. (2003) constataram teores de $\mathrm{F}$ de 160 e $126 \mathrm{mg} \mathrm{kg}^{-1} \mathrm{em}$ folhas de milho safrinha, semeado a 350 e $1.000 \mathrm{~m}$ de distância de indústria produtora de cerâmica.

Em poáceas, o sintoma de toxidez de flúor é geralmente caracterizado por clorose nas pontas e margens de folhas em desenvolvimento, estendendose às áreas internervais que se tornam necróticas.

O presente trabalho tem o objetivo de avaliar a influência do elemento flúor no desenvolvimento da cana-de-açúcar e o potencial de redução de produtividade quando da exposição a fontes emissoras de $\mathrm{F}$.

\section{Material e Métodos}

O estudo foi realizado em duas áreas - uma próxima da indústria produtora de cerâmica e outra distante $13.000 \mathrm{~m}$ - ambas no município de Cordeirópolis, Estado de São Paulo. A área ao lado da cerâmica é ocupada com as variedades de canade-açúcar SP80 1816 e RB83 5089, enquanto a distante, somente com a variedade SP80 1816. O relevo da região é suavemente ondulado, e as duas áreas possuem tipos de solos semelhantes, de textura argilosa. Nas duas áreas, o plantio foi realizado em outubro de 2002, o primeiro corte em setembro de 2003 e o segundo em outubro de 2004. O canavial foi queimado e colhido manualmente.

As práticas corretivas, adubação de plantio e de soqueira foram iguais nas duas áreas, assim como ausência de pragas de solo. Na adubação de plantio foram fornecidos 25,120 e $120 \mathrm{~kg} \mathrm{ha}^{-1}$ de $\mathrm{N}, \mathrm{P}_{2} \mathrm{O}_{5}$ e $\mathrm{K}_{2} \mathrm{O}$, respectivamente, quantidades suficientes para obtenção de altas produtividades de cana. $\mathrm{Na}$ adubação da primeira soqueira foram fornecidos 90 $\mathrm{kg} \mathrm{ha}^{-1}$ de $\mathrm{N}$ e de $\mathrm{K}_{2} \mathrm{O}$, quantidades suficientes para produtividades da ordem de $90 \mathrm{Mg} \mathrm{ha}^{-1}$, conforme recomendações de boletins oficiais de adubação (RAIJ et al., 1996). A área não recebeu aplicação de vinhaça.

Em abril de 2004, aos sete meses após o primeiro corte, foi realizada coleta de folhas pela técnica de diagnose foliar seguindo metodologia proposta por MALAVOLTA et al. (1997). Foram coletadas quatro amostras de folhas da seguinte maneira: para a variedade RB83 5089 foi retirada amostra composta a cerca de $80 \mathrm{~m}$ da indústria; para a variedade SP80 1816 foram coletadas folhas em três situações: a 20 m, $200 \mathrm{~m}$ e $13.000 \mathrm{~m}$ da cerâmica. As amostras foram remetidas para análises seguindo padrões técnicos: lavagem prévia com água destilada, detergente neutro e enxágüe com água destilada; secagem ao ar livre por 24 horas; secagem em estufa ventilada a $60{ }^{\circ} \mathrm{C}$ durante 48 horas. Após esse processo, o tecido vegetal foi finamente moído e encaminhado para análise. Segundo o método de FRANKENBERGER et al. (1996), o F foi extraído com ácido nítrico concentrado e determinado pelo método potenciométrico com eletrodo seletivo para F.

Visando avaliar a fertilidade do solo da área estudada para verificar a possibilidade da baixa produtividade ser devida a deficiências nutricionais, em abril de 2004 também foram coletadas amostras 
de solo do talhão próximo à cerâmica, contendo as duas variedades. O solo foi coletado com amostrador tipo sonda nas profundidades de 0 a 20 e 20 a $40 \mathrm{~cm}$, a $25 \mathrm{~cm}$ da linha da soqueira. Foram retiradas dez subamostras para compor cada amostra, que foram identificadas e encaminhadas para análise.

A produtividade de colmos das áreas avaliadas foi obtida pelo proprietário, mediante pesagem dos caminhões e medição da área colhida. Essa medida representa a estimativa da média de produtividade dos talhões observados.

\section{Resultados e Discussões}

\section{Solo}

Para os primeiros $20 \mathrm{~cm}$, os resultados foram: $\mathrm{pH}\left(\mathrm{CaCl}_{2}\right)=5,3 ; \mathrm{MO}=28 \mathrm{~g} \mathrm{dm}^{-3} ; \mathrm{P}_{\text {resina }}=14 \mathrm{mg} \mathrm{dm}^{-}$ 3. $\mathrm{S}=9 \mathrm{mg} \mathrm{dm}^{-3} ; \mathrm{K}=3,5 \mathrm{mmol}_{\mathrm{c}} \mathrm{dm}^{-3} ; \mathrm{Ca}=44 \mathrm{mmol}_{\mathrm{c}}$ $\mathrm{dm}^{-3} ; \mathrm{Mg}=15 \mathrm{mmol}_{\mathrm{c}} \mathrm{dm}^{-3} ; \mathrm{Al}=1 \mathrm{mmol}_{\mathrm{C}} \mathrm{dm}^{-3} ; \mathrm{CTC}=$ $87,5 \mathrm{mmol}_{\mathrm{c}} \mathrm{dm}^{-3}$ e saturação por bases $(\mathrm{V} \%)=71$. Para a camada de 20 a $40 \mathrm{~cm}$ os resultados foram semelhantes: $\mathrm{pH}\left(\mathrm{CaCl}_{2}\right)=5,4 ; \mathrm{MO}=28 \mathrm{~g} \mathrm{dm}^{-3} ; \mathrm{P}_{\text {resina }}$ $=10 \mathrm{mg} \mathrm{dm}^{-3} ; \mathrm{S}=6 \mathrm{mg} \mathrm{dm}^{-3} ; \mathrm{K}=1,9 \mathrm{mmol}_{\mathrm{c}} \mathrm{dm}^{-3} ; \mathrm{Ca}$ $=42 \mathrm{mmol}_{\mathrm{c}} \mathrm{dm}^{-3} ; \mathrm{Mg}=15 \mathrm{mmol}_{\mathrm{c}} \mathrm{dm}^{-3} ; \mathrm{Al}=0 \mathrm{mmol}_{\mathrm{c}}$ $\mathrm{dm}^{-3} ; \mathrm{CTC}=87 \mathrm{mmol}_{\mathrm{c}} \mathrm{dm}^{-3}$ e saturação por bases $(V \%)$ $=68$. Em relação aos micronutrientes, na camada até $20 \mathrm{~cm}$ os teores, em $\mathrm{mg} \mathrm{dm}^{-3}$, foram de: $\mathrm{B}=0,9 ; \mathrm{Cu}=$ 1,$3 ; \mathrm{Fe}=19 ; \mathrm{Mn}=18$ e $\mathrm{Zn}=0,8$. Para a camada de 20 a $40 \mathrm{~cm}$, os teores também foram semelhantes: $\mathrm{B}=0,9$; $\mathrm{Cu}=1,4 ; \mathrm{Fe}=11 ; \mathrm{Mn}=19$ e $\mathrm{Zn}=0,5$. O boro foi extraído pelo método da água quente e os demais pelo método do DTPA.

O solo possui saturação por bases adequada, baixa acidez, baixo teor de alumínio tóxico e relação adequada de cálcio, magnésio e potássio na CTC. O teor de $\mathrm{Zn}$ ficou abaixo do adequado, segundo RAIJ et al. (1996). O solo da área estudada é, portanto, de fertilidade adequada para o cultivo de cana-de-açúcar, e capaz de proporcionar altas produtividades quando os demais fatores de produção forem adequados.

\section{Planta}

$\mathrm{Na}$ amostra de folha retirada a $80 \mathrm{~m}$, constatam-se teores discrepantes de $\mathrm{K}$ e Ca em relação às demais provavelmente por se tratar de outra variedade (Tabela 1).

O teor de nitrogênio está abaixo da faixa considerada adequada para todas as amostras, conforme SPIRONELlo et al (1996). O teor de fósforo é considerado adequado em todas as amostras e decresce linearmente à medida que se afasta da cerâmica, e pode estar ocorrendo efeito de concentração desse nutriente no tecido foliar (próximo à cerâmica pela menor produção de matéria seca). Esse efeito parece ocorrer também para o potássio, especialmente na variedade RB83 5089, em que se observou maior produtividade e menor teor de K no tecido foliar. Os teores de cálcio, magnésio e enxofre estão dentro da faixa considerada adequada para todas as amostras.

O teor de flúor no tecido vegetal decresce linearmente à medida que se afasta da indústria de cerâmica (Tabela 2). Esses teores variam de 56 (a 20 m) a $3 \mathrm{mg} \mathrm{kg}^{-1}$ (cerca de $13.000 \mathrm{~m}$ de distância) para a variedade SP80 1816. A $80 \mathrm{~m}$ da área de influência da cerâmica, o teor foi de $26 \mathrm{mg} \mathrm{kg}^{-1}$ para a variedade RB83 5089.

Esses teores estão acima dos considerados normais, de $10 \mathrm{mg} \mathrm{kg}^{-1}$ ou menos, segundo GARBER at al. (1967) e outros pesquisadores, citados em ARNDT et al. (1995), em áreas sem influência de emissão de F. Em ambas as variedades, os teores de F observados nas plantas próximas à cerâmica causaram sintomas de toxidez. Na região mais próxima da indústria, o desenvolvimento foi severamente comprometido a ponto de inviabilizar a colheita. À medida que se afasta da fonte emissora de flúor, os sintomas de toxidez diminuem de intensidade. $\mathrm{Na}$ amostra coletada a $13.000 \mathrm{~m}$ da cerâmica, o teor de flúor no tecido vegetal $\left(3 \mathrm{mg} \mathrm{kg}^{-1}\right)$ é considerado normal e não foram evidenciados sintomas de toxidez, sem causar interferência no desenvolvimento da cana-de-açúcar.

Tabela 1. Teores de macronutrientes no tecido vegetal a distâncias variadas da cerâmica

\begin{tabular}{|c|c|c|c|c|c|c|c|}
\hline Distância & Variedade & $\mathrm{N}$ & $\mathrm{P}$ & K & $\mathrm{Ca}$ & $\mathrm{Mg}$ & S \\
\hline $\mathrm{m}$ & & 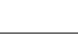 & . & 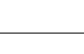 & & & \\
\hline 20 & SP80 1816 & 16,0 & 2,7 & 17,1 & 2,2 & 1,5 & 1,5 \\
\hline 80 & RB83 5089 & 16,7 & 2,4 & 10,2 & 5,4 & 2,4 & 1,6 \\
\hline 200 & SP80 1816 & 14,8 & 2,0 & 12,0 & 3,6 & 2,3 & 1,7 \\
\hline 13.000 & SP80 1816 & 15,7 & 1,9 & 12,8 & 3,8 & 1,9 & 1,6 \\
\hline
\end{tabular}


Tabela 2. Teores de flúor e micronutrientes no tecido vegetal a distâncias variadas da cerâmica

\begin{tabular}{|c|c|c|c|c|c|c|c|}
\hline Distância & Variedade & $\mathrm{F}$ & B & $\mathrm{Cu}$ & $\mathrm{Fe}$ & Mn & $\mathrm{Zn}$ \\
\hline $\mathrm{m}$ & & & & 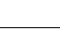 & & & \\
\hline 20 & SP80 1816 & 56 & 10 & 7 & 306 & 54 & 21 \\
\hline 80 & RB83 5089 & 26 & 6 & 8 & 350 & 139 & 28 \\
\hline 200 & SP80 1816 & 14 & 4 & 6 & 492 & 68 & 17 \\
\hline 13.000 & SP80 1816 & 3 & 4 & 7 & 362 & 51 & 19 \\
\hline
\end{tabular}

Quanto aos micronutrientes, os teores de boro observados nas folhas foram baixos, com possível ocorrência de diluição da concentração nas amostras mais distantes da cerâmica. Os teores de cobre e zinco estão dentro da faixa considerada adequada e os de ferro acima. Na variedade RB83 5089, verificaram-se elevados teores de manganês no tecido vegetal (Tabela 2).

Os resultados de análises de folhas obtidas pela técnica de diagnose foliar diferem bastante em função da variedade e distância entre local da amostragem e fonte emissora de flúor, indicando interferência desse elemento $(F)$ no metabolismo da planta, seja na absorção, transporte ou redistribuição dos demais nutrientes na cana-de-açúcar.

A sensibilidade das plantas à contaminação atmosférica com $\mathrm{F}$ é dependente da espécie e da variedade. Em algumas espécies tolerantes, como algodão (Gossypium hirsutum), alfafa (Medicago sativa), chá (Camellia sinensis) e Manacá-da-Serra (Tibouchina pulchra), podem não ocorrer sintomas visuais de toxidez mesmo com concentrações foliares acima de $200 \mathrm{mg} \mathrm{kg}^{-1}$ (Thomas, 1965; Arndt, 1995; Cepa, 1996; KLumPP et al., 1998). Por outro lado, algumas espécies da família das poáceas são sensíveis à presença de $\mathrm{F}$ atmosférico, como é o caso do milho (Zea mays) e do capim-colonião (Panicum maximum). A cana-de-açúcar é geralmente classificada no grupo sensível ou intermediário (ARNDT et al., 1995).

\section{Produtividade}

A produtividade de colmos da cana-planta foi de $54 \mathrm{Mg} \mathrm{ha}^{-1}$ para as duas variedades na área sob influência do F e de $82 \mathrm{Mg} \mathrm{ha}^{-1}$ na área sem influência do F para a variedade SP80 1816.

Na primeira soqueira, a produtividade foi de $50 \mathrm{Mg} \mathrm{ha}^{-1}$ para a variedade SP80 1816 e $60 \mathrm{Mg} \mathrm{ha}^{-1}$ para a RB83 5089 na área sob influência do F, indicando ser essa variedade é mais tolerante ao $\mathrm{F}$, enquanto na área sem influência do $\mathrm{F}$ a produtividade foi de $100 \mathrm{Mg} \mathrm{ha}^{-1}$.

Nem sempre é possível definir os níveis de toxicidade de F que causam prejuízos ao rendimento das culturas em razão da contaminação aérea abranger grandes áreas nas quais outros fatores, tais como variações de solo, condições climáticas (precipitação pluvial, temperatura e umidade), manejo da cultura entre outros, podem interferir na produtividade. Em alguns casos, o dano se restringe à área necrosada, que deixa de fazer fotossíntese, mas os prejuízos podem ser reduzidos se a perda de tecido for compensada pelo aumento da taxa de fotossíntese ou crescimento em outros tecidos (WEINSTEIN et al., 1998).

Embora os dados de rendimento deste estudo tenham sido estimados sem os controles convencionais de áreas experimentais e possa haver variações de solo e de precipitação pluvial entre os talhões avaliados, os sintomas visuais e os altos teores de $\mathrm{F}$ verificados nas folhas revelam que a toxidez de $\mathrm{F}$ tenha tido estreita relação com a queda de produtividade da cana-de-açúcar nas áreas próximas à cerâmica.

\section{Conclusão}

Os teores de flúor (F) encontrados na folha de plantas localizadas próximo a indústria produtora de cerâmica estão acima dos considerados normais e pode ser associado à queda na produtividade da cultura. A cana-de-açúcar é sensível à absorção excessiva de F.

\section{Agradecimentos}

Agradecimento a Valmir Tomazella por ceder a área para os estudos e aos integrantes do Grupo de Apoio à Pesquisa e Extensão pelo apoio na elaboração do trabalho.

\section{REFERÊNCIAS}

ARNDT, U.; FLORES, F.; WEINSTEIN, L. Efeitos do flúor sobre as plantas: diagnose de danos na vegetação do Brasil. 1.ed. Porto Alegre: Editora da Universidade/UFRGS, 1995. 154p. 
BREWER, R.F.; GUILLEMET, F.B.; SUTHERLAND, F.H. The effects of atmospheric fluoride on gladiolus growth, flowering and corm production. Proceedings of the American Society for Horticultural Science, Alexandria, v.88, p.634-644, 1965.

CEPA/FPAC. National Ambient Air Quality Objectives of Hydrogen Fluoride (HF). Science Assessment Document. Report by the CEPA/FPAC Working Group on Air Quality Objectives and Guidelines. Toronto: Canadian Environmental Protection ACT, 1996. 104p.

COMPANHIA DE TECNOLOGIA DE SANEAMENTO AMBIENTAL - CETESB. Projeto de prevenção à poluição em indústrias de pisos e revestimentos cerâmicos. São Paulo: CETESB, 1998. Acesso em: 15 jan. 2005. Disponível em: http:/ / www.cetesb.sp.gov.br/Ambiente/prevencao_poluicao/ download/ceramica.pdf.

FRANKENBERGER, W.T.; TABATABAI, M.A.; ADRIANO, D.C.; DONER, H.E. Bromine, Chlorine and Fluorine. In: Methods of Soil Analysis: Chemical Methods. Part 3. Madison, Wisconsin,USA: SSSA, 1996. p.847-867.

FORTES, C.; DUARTE, A.P.; MATSUOKA, S.; HOFFMANN, H.P.; LAVORENTI, N.A. Toxicidade de flúor em cultivares de milho em área próxima a uma indústria cerâmica, Araras (SP). Bragantia, Campinas, v.62, p.275-281, 2003.

KLUMPP, A.; DOMINGOS, M.; MORAES, RM.M. de; KLUMPP, G. Effects of complex air pollution on tree species of the Atlantic rain Forest near Cubatão, Brazil. Chemosphere, Oxford, v.36, p.989-994, 1998.
MALAVOLTA, E.; VITTI, G.C.; OLIVEIRA, S.A. Avaliação do estado nutricional das plantas: princípios e aplicações. 2.ed. Piracicaba: POTAFÓS, 1997. p.115-230.

RAIJ, B. van; QUAGGIO, J.A; CANTARELLA, H.; ABREU, C.A. Interpretação de resultados de análises de solo. In: RAIJ, B. van; CANTARELLA, H.; QUAGGIO, J.A.; FURLANI, A.M.C. Recomendações de adubação e calagem para o Estado de São Paulo. 2.ed. Campinas: Instituto Agronômico/Fundação IAC, 1996. p.8-13. (Boletim Técnico 100)

SPIRONELLO, A.; RAIJ, B. van; PENATTI, C.P.; CANTARELLA, H.; MORELLI, J.L.; ORLANDO FILHO, J.; LANDELL, M.G. de A.; ROSSETO, R. Outras culturas industriais. In: RAIJ, B. van.; CANTARELLA, H.; QUAGGIO, J.A.; FURLANI, A.M.C. Recomendações de adubação e calagem para o Estado de São Paulo. 2.ed. Campinas: Instituto Agronômico/ Fundação IAC, 1996. p.233-239. (Boletim Técnico 100)

THOMAS, M.D. Gas damage to plants. Annual Review of Plant Physiology, Palo Alto, v.2, p.293-332, 1965.

WEINSTEIN, L.H.; DAVISON, A.W.; ARNDT. U. Fluoride. In FLAGER, R.B. (Ed.) Recognition of air pollution injury by vegetation; a pictorial atlas. 2.ed. Pittsburgh: Air and Waste Management Association, 1998. 